\title{
A redução do gradiente na via de saída do ventrículo esquerdo pelo marcapasso DDD em pacientes com miocardiopatia hipertrófica obstrutiva
}

\author{
Farid César FAES, João Ricardo SANT’ANNA, Paulo Roberto PRATES, Renato A.K. KALIL, Ivo A. NESRALLA
}

RBCCV 44205-597

\section{Resumo}

Objetivo: A estimulação seqüencial atrioventricular (modo DDD) tem sido aceita como uma terapêutica eficaz para reduzir o gradiente na via de saída do ventrículo esquerdo (GVSVE) em pacientes com miocardiopatia hipertrófica obstrutiva (MHO).

O objetivo deste trabalho é avaliar o efeito do marcapasso DDD para redução do GVSVE em pacientes com MHO de má resposta ao tratamento farmacológico e com obstrução na via de saída ventricular esquerda com gradiente crescente ou superior a $50 \mathrm{mmHg}$.

Método: Um marcapasso de dupla câmara, composto de gerador de pulsos DDD e dos eletrodos atrial e ventricular, foi implantado mediante técnica transvenosa em 42 pacientes no período de fevereiro de 1995 a março de 2001 . Foi observada uma redução na sintomatologia e uma melhora na classe funcional na maioria dos pacientes.

Resultados: Quanto à classe funcional, na última avaliação, 31 pacientes estavam em classe funcional $I$, e 10 pacientes na classe funcional II, contrastando com o pré-operatório, quando 30 pacientes estavam na classe funcional III ou IV, e apenas 12 estavam na classe II (p<0,001). A medicação foi ajustada, sendo reduzida ou suspensa em 15 pacientes, substituída em 3 pacientes e aumentada (quanto ao número de fármacos ou sua dose) em 14 pacientes. O GVSVE reduziu significativamente de $96,50 \pm 30,55 \mathrm{mmHg}$ para $41,80 \pm 22,84 \mathrm{mmHg}$ após ajuste do aparelho $(\mathbf{p}<0,001)$.

Conclusão: Após o implante do marcapasso DDD existe uma melhora clínica e ecocardiográfica em pacientes com MHO de má resposta ao tratamento clínico. Os resultados obtidos permitem a continuidade do uso da terapêutica em casos selecionados.

Descritores: Miocardiopatia hipertrófica obstrutiva. Marcapasso. Cirurgia.

\section{Abstract}

Objective: Dual chamber pacing has been recognized as an complementary therapy to reduce left ventricular outflow tract gradient (LVTOG) in patients with hypertrophic obstructive cardiomyopathy (HOCM). Although controversies still remains, most reports show the effectiveness of this novel method to reduce LVOTG, improved clinical status and increase survival in patients with HOCM refractary to clinical treatment. The aim of this study is to present the results of DDD pacemaker therapy in a group of patients with HOCM unresponsive to pharmacological management.

Method: Forty-two patients with HOCM refractory to medical treatment and with an increasing or higher than $50 \mathrm{mmHg}$ LVOTG were implanted a transvenous DDD pacemaker. The optimal atrioventricular interval was defined as that producing the lowest left ventricular outflow tract gradient without compromise of aortic or left atrial pressures. After implantation, the pacemaker was programmed with a pulse rate capable of controlling the atria and the ventricle with a reduced atrioventricular interval (equal or lower than $120 \mathrm{~ms}$ ). Pacemaker programming was performed during ECHO evaluation to obtain the lowest LVOTG.

Results: The majority of patients showed hemodynamic improvement during permanent pacing. Initial pacemaker programming resulted in a reduction of the LVOTG from the mean control value of $96.50 \pm 30.55 \mathrm{mmHg}$ to $41.80 \pm 22.84 \mathrm{mmHg}$ $(p<0,001)$, and in the last follow-up LVTOG decreased even further to $30.00 \pm 23.03$.

Conclusion: Dual-chamber pacing improve subjective measures of functional status in patients with symptomatic HOCM in this study, offering reduction in LVOTG and improvements in objective measures of patient symptoms and functional status.

Descriptors: Hypertrophic obstructive cardiomyopathy. Pacemaker, treatment. 


\section{INTRODUÇÃO}

As características específicas da miocardiopatia hipertrófica obstrutiva foram descritas há mais de 100 anos ${ }^{(1-4)}$, mas o seu estudo sistemático iniciou no final dos anos $50^{(5,6)}$. Àquela época se identificou a principal característica da doença: gradiente de pressão dinâmica subaórtica, dividindo o ventrículo esquerdo em uma região apical de pressão elevada e em uma outra área subaórtica de menor pressão. A patologia ainda se caracteriza por uma rigidez anormal das paredes do ventrículo esquerdo na diástole, criando dificuldades para o enchimento diastólico, causando elevação da pressão telediastólica do ventrículo esquerdo, resultando em congestão pulmonar e em dispnéia, sintomas comuns da doença.

Embora muitos pacientes respondam à terapêutica farmacológica, a doença pode progredir com a dilatação ventricular progressiva e com a insuficiência cardíaca congestiva ou conduzir à morte súbita.

A prevalência da miocardiopatia hipertrófica é de 1 por $500^{(5)}$ na população em geral. No estudo de Framingham, dentre os pacientes estudados com ecocardiografia, a anormalidade estava presente em $0,3 \%$ da população com idade média de 47 anos, e aumentava para $1,7 \%$ na população com idade média de 74 anos, ${ }^{(6)}$ isto porque, sendo dependente da idade, pode desenvolver-se com o passar dos anos.

A doença é geneticamente transmitida em mais de 50\% dos pacientes tendo padrão autossômico dominante de genes com expressão e penetrância variáveis. Também pode ser possível que genes não-sarcoméricos causem a miocardiopatia hipertrófica.

Uma das características mais notáveis da doença é a sua heterogeneidade fenotípica, ${ }^{(7-9)}$ que pode ser causada em pelo menos dois loci e isso foi visto a partir da existência de um gene localizado no braço largo do cromossoma 14, na banda mais próxima do centrômero e foi denominado FHC1. Descobriu-se posteriormente que este gen codifica a cadeia pesada de miosina. ${ }^{(10)}$

A maioria dos casos tem uma herança autossômica dominante, ${ }^{(11)}$ e nesses casos, a penetrância é relacionada à idade, incompleta e variável. A penetrância incompleta e a dificuldade de definição dos sinais e exames têm dificultado a compreensão dessa patologia.

A cardiomiopatia hipertrófica familiar é provavelmente a doença cardiovascular que com mais frequiência se transmite por anomalias genéticas. ${ }^{(12)}$

A miocardiopatia hipertrófica obstrutiva (MHO) está caracterizada por uma função sistólica global hiperdinâmica. (13) Embora os parâmetros clínicos da ejeção ventricular esquerda, como a fração de ejeção estejam elevados, a contratilidade ventricular não é necessariamente elevada nessa doença. Como as paredes são espessadas e o volume da cavidade é diminuído, o aparente acréscimo na função sistólica pode ser apenas um reflexo de condições alteradas do enchimento ventricular.

A obstrução dinâmica da via de saída do ventrículo esquerdo, observada na MHO é diferente da obstrução da doença valvar aórtica em três aspectos: 1) a queda de pressão ocorre dentro do ventrículo esquerdo e não através da válvula; 2) a obstrução é dinâmica, o gradiente de pressão pode ser variável, ao contrário do grau de obstrução relativamente fixo na doença valvar aórtica e 3) manobras que reduzem a pré-carga aumentam a obstrução muscular subaórtica, reduzindo o volume da cavidade e o tamanho da via de saída.

Na MHO existe um gradiente dinâmico de pressão, que parece estar relacionado a um estreitamento adicional da via de saída estenosada pela hipertrofia septal e dinâmica alterada da valva mitral (movimento sistólico anterior). A maior parte dos investigadores acredita que haja fatores mecânicos obstrutivos à ejeção do ventrículo esquerdo e que sejam o resultado da assinergia da valva mitral, onde faz contato com o septo interventricular na mesosístole, talvez pelo efeito Venturi ou pelo aumento da velocidade de expulsão produzidos pela orientação e geometria anormal naquela área.

Os pacientes com miocardiopatia hipertrófica mostram, ainda, algum grau de disfunção diastólica independente dos sintomas e do GVSVE. A disfunção diastólica produz maior pressão de enchimento e pode ser o resultado da desarmonia entre o relaxamento e a distensibilidade ventricular esquerda. O enchimento diastólico inicial está prolongado quando se inicia o relaxamento e isso pode ser associado à cinética anormal do cálcio, à isquemia subendocárdica ou a condições anormais de carga telediastólica. O enchimento telediastólico é alterado quando se perturba a distensibilidade do ventrículo esquerdo, aumentando a pressão de enchimento. A miocardiopatia hipertrófica causa distensibilidade anormal devido à fibrose e/ou à desorganização celular.

A isquemia miocárdica é freqüente e multifatorial, ${ }^{(14)}$ ocorrendo mesmo em casos onde não se demonstrou a coronariopatia arteriosclerótica obstrutiva. A angina pode ser atípica, de duração prolongada ao repouso, sem relação com o exercício e não aliviando com nitratos. A isquemia regional pode ocorrer e é responsável pela precordialgia em muitos pacientes. As causas da isquemia são: 1) aumento da demanda de oxigênio do miocárdio, conforme evidenciada em estudo com marcapasso atrial, quando a precordialgia é precipitada. Isso pode estar associado a uma reduzida resposta vasodilatadora, ao aumento da pressão de enchimento e anormalidades no metabolismo do lactato, o que pode levar ao desenvolvimento de metabolismo aneróbico; 2) defeitos de perfusão miocárdica induzidos pelo exercício, de acordo com aquisição de imagem radioisotópica cardíaca e 3) melhora da função regional do ventrículo esquerdo com o repouso. Esses achados são compatíveis com a isquemia regional prévia e com cicatrização do ventrículo esquerdo, extensas áreas de fibrose e de cicatrizes 
transmurais. A isquemia miocárdica provocada pelo esforço pode ocorrer se a demanda miocárdica por oxigênio for excessiva e se ultrapassar a capacidade de suprimento miocárdio pelas coronárias normais. A demanda supranormal de oxigênio parece resultar, pelo menos em parte, do aumento da massa muscular e de altas pressões na via de saída obstrutiva do ventrículo esquerdo.

Os mecanismos que levam ao desenvolvimento do desequilíbrio entre a oferta e a demanda incluem, ainda, a densidade inadequada de capilares no músculo hipertrófico e a presença de estreitamento intramural das coronárias intramiocárdicas.

Desde que inicia o desenvolvimento da isquemia, pode ocorrer um aumento na já anormalmente elevada pressão de enchimento do ventrículo esquerdo, predispondo à maior isquemia. A extração de oxigênio diminuída pode, também, contribuir ou exacerbar a isquemia.

\section{Sintomas}

O quadro clínico varia desde a ausência de sintomas até o aparecimento de sintomas incapacitantes. A maioria dos pacientes é assintomática ou levemente sintomática. A primeira manifestação pode ser a morte súbita. A hiperpertrofia ventricular esquerda, o gradiente de pressão do ventrículo esquerdo, a disfunção diastólica e a isquemia miocárdica em seus diversos graus são os determinantes da variável sintomatologia referida por diferentes pacientes.

O sintoma mais freqüente é a dispnéia, que se apresenta em até $90 \%$ dos pacientes sintomáticos e é, em grande parte, conseqüência da elevada pressão diastólica do ventrículo esquerdo, resultado da alteração do enchimento ventricular pela disfunção diastólica. ${ }^{(15)}$ São freqüentes a angina (75\% dos pacientes), o cansaço, a pré-síncope e a síncope. Estão também presentes: a palpitação, a dispnéia paroxística noturna, a insuficiência cardíaca congestiva e as tonturas. O exercício exacerba os sintomas. ${ }^{(16)}$

A angina se deve em parte ao desequilíbrio entre o aporte e a demanda de oxigênio como conseqüência de uma massa miocárdica muito aumentada. $\mathrm{O}$ infarto transmural pode ocorrer na ausência de estreitamento de coronárias extramurais. ${ }^{(17)}$

A síncope pode ocorrer por consumo excessivo de oxigênio cardíaco ou por arritmia. É mais freqüente em pacientes jovens com um ventrículo esquerdo de cavidade pequena e que mostram taquicardia ventricular. As crises de pré-síncope ocorrem em bipedestação e aliviam com o repouso. Os pacientes jovens com pré-síncope e com síncope são os mais sujeitos à morte súbita. ${ }^{(18)}$

O implante de marcapasso de dupla câmara é, atualmente, um procedimento universalmente aceito para o tratamento da miocardiopatia hipertrófica septal obstrutiva refratária ao tratamento com drogas e uma alternativa às terapêuticas cirúrgicas como miomectomia septal ou substituição da valva mitral.

O implante do marcapasso para o tratamento da miocardiopatia hipertrófica foi especulado nos anos 70. Os primeiros conceitos foram propostos na Alemanha. ${ }^{(19)}$ Mas, na década de 90 , surgiu um maior número de estudos com evidências dos benefícios do marcapasso bicameral ${ }^{(20)}$. A idéia que embasa o procedimento é de que a ativação precoce da porção apical do septo interventricular alteraria o seu padrão de despolarização, diminuindo, ou mesmo abolindo o contato do septo interventricular com o folheto anterior da valva mitral. Para uma melhor obtenção do benefício, é importante, ou mesmo crucial, a manutenção adequada do intervalo atrioventricular. A perfeita adequação do intervalo A-V depende de dois fatores: 1) o intervalo A$\mathrm{V}$ deve ser menor do que o do paciente quando em ritmo sinusal; 2) o enchimento ventricular deve ser otimizado e, isso deve ser atingido com a adequada programação do intervalo A-V que suprime o ritmo próprio do paciente sem alterar ou mesmo melhorando o enchimento ventricular. Com a necessidade de se manter o marcapasso funcionando sempre parece lógico supor que o benefício maior seria para os pacientes que apresentem intervalos A-V longos, portanto, o uso de drogas com cronotropismo negativo deve ser mantido de modo a não comprometer o enchimento diastólico. ${ }^{(21)}$

Foi demonstrado que o marcapasso DDD reduz a obstrução do fluxo de saída no ventrículo esquerdo, melhorando os sintomas. O ventrículo direito altera, de forma aguda, o padrão de contração do ventrículo esquerdo que pode aumentar as dimensões da via de saída sistólica e, por conseguinte, reduzir a velocidade sangüíneas do trato de saída do ventrículo esquerdo, diminuindo o movimento sistólico anterior, reduzindo a obstrução da via de saída do ventrículo esquerdo e a regurgitação mitral associada. $\mathrm{O}$ ritmo prolongado também altera as propriedades elétricas e hemodinâmicas do miocárdio.

Cerca de $25 \%$ dos doentes têm um gradiente elevado na câmara de saída do ventrículo esquerdo e, desse gradiente dependem a massa, a rigidez ventricular e, de alguma forma, a sintomatologia do paciente. E é no período pós-esforço imediato $\left(3^{\circ}\right.$ e $5^{\circ}$ minuto de recuperação) que se encontram aumentos mais significativos do gradiente. Isso se dá, provavelmente, à diminuição do retorno venoso e, em conseqüência, há aumento da obstrução da câmara de saída do ventrículo esquerdo.

Embora ainda não exista uma resposta definitiva quanto ao mecanismo ou aos mecanismos responsáveis pela melhora hemodinâmica, o que se sabe é que o estímulo ventricular direito provoca um movimento paradoxal do septo interventricular. Esse movimento aumenta a capacidade volumétrica da via de saída do ventrículo esquerdo e, ao mesmo tempo, reduz a velocidade do sangue naquele local, com menor movimento sistólico anterior da valva mitral e, dessa maneira, diminui a regurgitação mitral.

Efeito do marcapasso de dupla câmara no gradiente da via de saída do ventrículo esquerdo

O gradiente é causado pelo septo hipertrofiado que se 
projeta na via de saída do ventrículo esquerdo durante a sístole e é acrescido do movimento sistólico anterior do folheto anterior da valva mitral. $\mathrm{O}$ deslocamento de todo o aparelho valvar mitral também pode contribuir para a obstrução. O início da contração originada do ápice do ventrículo direito pode alterar a contração septal e, com isso, interromper a seqüência fisiopatológica dos eventos, causando uma diminuição no gradiente. Embora a grande maioria dos pacientes tenha redução significativa no gradiente, em alguns casos, não ocorrem mudanças e, em outros, pode até haver aumento desse gradiente. $\mathrm{O}$ aumento do gradiente pode ser pelo enchimento inadequado do ventrículo esquerdo e/ou pela contração atrial inefetiva. $\mathrm{O}$ gradiente é dependente da contratilidade sistólica e das condições de enchimento do ventrículo esquerdo, podendo ser muito variável nas diferentes condições dinâmicas e durante períodos diferentes.

Efeito do marcapasso de dupla câmara na função sistólica do ventrículo esquerdo

O marcapasso deve ter um programa adequado para a melhor sequiência átrio-ventricular, otimizando o enchimento e a contração do ventrículo esquerdo. A contração atrial, no fim da diástole, permite a manutenção de pressões atriais baixas durante a fase inicial e médias da diástole enquanto aumenta a pré-carga imediatamente antes da contração ventricular. Se o intervalo átrio-ventricular é encurtado demais, haverá uma pré-carga menor no início da contração ventricular, e o volume de ejeção será menor. O efeito é mais evidente quando o intervalo AV é menor que $60 \mathrm{~ms}$, com diminuição significativa do rendimento cardíaco.

Efeito do marcapasso de dupla câmara na função diastólica do ventrículo esquerdo

O enchimento diastólico do ventrículo esquerdo é uma sequiência complexa, e é difícil isolar os vários componentes em um coração intacto. O relaxamento ventricular está deteriorado na miocardiopatia hipertrófica e contribui para o aumento das pressões de enchimento. As razões que afetam negativamente o relaxamento muscular incluem: 1) uma carga contrátil aumentada imposta ao ventrículo esquerdo pela obstrução da via de saída com o relaxamento anormal e um enchimento coronariano diminuído; 2) desativação anormal dos sítios geradores de energia causada por isquemia e sobrecarga de cálcio e, 3) desuniformidade no enchimento e desativação no tempo e no espaço. ${ }^{(22)}$

\section{MÉTODO}

Este estudo é do tipo longitudinal prospectivo, compreendendo uma coorte de pacientes com MHO que foram tratados mediante marcapasso DDD e acompanhados com vistas a se obter redução na obstrução da VSVE.

Previamente ao implante, os pacientes receberam esclarecimentos quanto ao tratamento proposto para a sua doença, as limitações inerentes ao marcapasso, a necessidade de acompanhamento periódico, os resultados já obtidos na literatura e as alternativas de tratamento existentes. Após a obtenção do consentimento, o procedimento foi efetuado.

O protocolo de estudo foi cadastrado na Unidade de Pesquisa e contou com aprovação do Comitê de Ética em Pesquisa, do Instituto de Cardiologia do Rio Grande do Sul.

A indicação para o implante do marcapasso consistiu na intolerância ou na refratariedade ao tratamento clínico, no elevado gradiente sistólico instantâneo máximo na via de saída do ventrículo esquerdo (GSVSVE) ou no seu progressivo aumento com valores superiores a $50 \mathrm{mmHg}$ ao ecocardiograma bidimensional com Doppler.

No ecocardiograma imediatamente anterior ao implante do marcapasso, o GVSVE foi de $96,50 \pm 30,55 \mathrm{mmHg}$.

No período entre fevereiro de 1995 e março de 2001, 42 pacientes com MHO implantaram um marcapasso DDD, buscando reduzir a obstrução na VSVE. Dos pacientes, 26 eram do sexo feminino $(61,9 \%)$ e 16 masculinos $(38,1 \%)$, e sua idade variava entre 4 anos e 77 anos (média de 44,56 anos). (Figura 1).

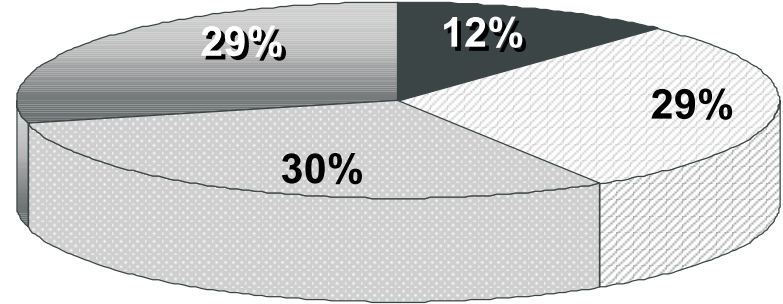

até 20 anos $\square$ 21-40 $\square$ 41-60 $\square$ acima de 60

Fig. 1 - Idade

A sintomatologia predominante consistia em angina (19 pacientes: $45,24 \%$ ), dispnéia (13 pacientes: $30,9 \%$ ); cansaço (18 pacientes: $42,86 \%$ ); palpitação (4 pacientes: 9,5\%) ou tonturas e/ou lipotímia (20 pacientes: 47,62\%) (Figura 2).

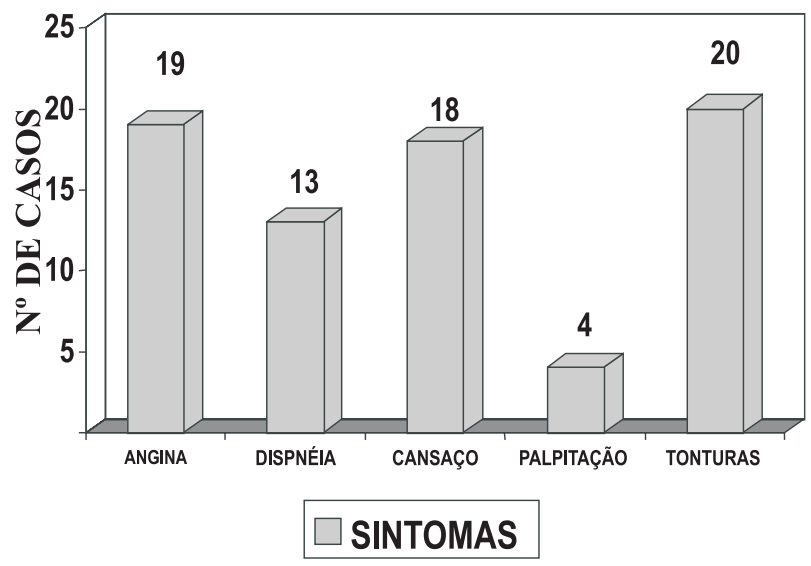

Fig. 2 - Sintomatologia pré-operatória 
Um dos pacientes era assintomático, mas era o paciente de 4 anos, que por razões óbvias, não verbalizava a sintomatologia. A classe funcional pré-operatória, de acordo com a New York Heart Association, está representada na Figura 3.

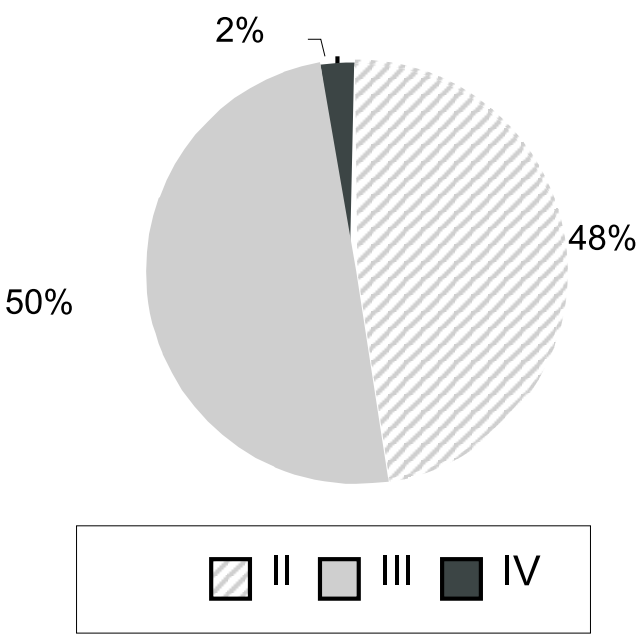

Fig. 3 - Classe funcional no pré-operatório

Os pacientes faziam uso de medicação do tipo betabloqueador (29 pacientes: 69\%); antagonistas do cálcio (15 pacientes: $35,7 \%$ ); disopiramida (5 pacientes: $11,9 \%$ ) ou a sua associação (19 pacientes: 45,2\%); 6 pacientes $(14,2 \%)$ não usavam medicação (Figura 4).

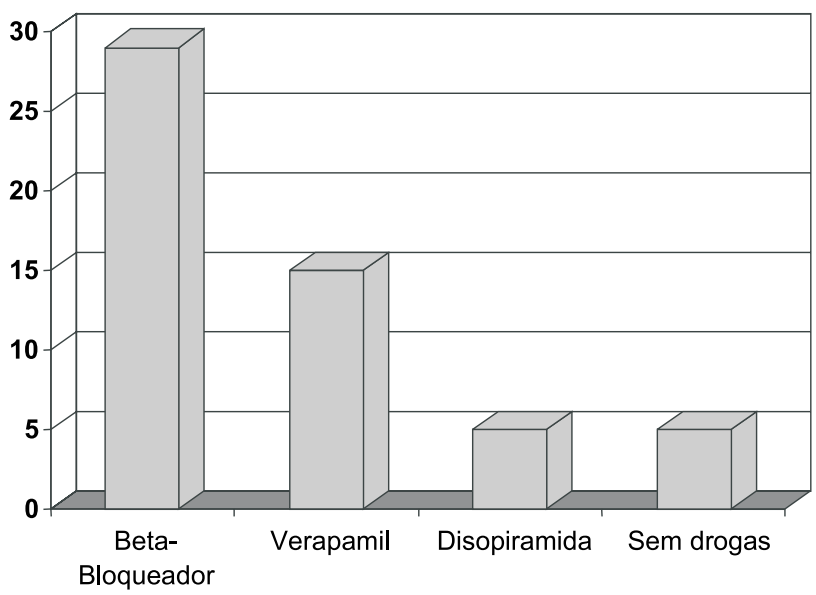

Fig. 4 - Drogas no pré-operatório

\section{Cuidados Pré-Operatórios}

Previamente ao tratamento proposto, foi colocado aos pacientes o procedimento adequado para a doença, as técnicas compreendidas, as limitações inerentes ao procedimento, a necessidade de acompanhamento periódico, os resultados já obtidos na literatura e as alternativas de tratamento existentes. Após a concordância do paciente quanto ao implante do marcapasso, o procedimento foi realizado.
Durante a internação hospitalar e antes do implante do marcapasso, os pacientes foram submetidos a estudo ecocardiográfico bidimensional com Doppler em cores, visando confirmar a obstrução na VSVE devido a MHO. Um período de jejum de 6 horas foi mantido e medicação pré-anestésica não foi prescrita antes do procedimento.

\section{Técnica de Implante do Marcapasso}

Para o implante de marcapasso, foi utilizada monitorização eletrocardiográfica e oximetria de pulso com um acesso venoso periférico. Nos pacientes adultos foi colocado um cateter nasal para a administração de oxigênio com o fluxo de 2 litros por minuto e durante a cirurgia a sedação foi conduzida com midazolan e fentanila. Realizada a assepsia da pele com degermante e álcool iodadado seguido da colocação de campos esterelizados. O local da incisão e da loja foi infiltrado com solução de lidocaína a $1 \%$. A técnica de inserção transvenosa foi a escolhida e o sulco deltopeitoral direito de preferência foi dissecado e a veia cefálica reparada. Quando a veia foi considerada inadequada para a introdução de um ou os dois eletrodos a punção da veia subclávia foi a opção seguinte.Os eletrodos atriais e ventriculares foram avançados até o coração e fixados sob controle fluoroscópico. O eletrodo ventricular foi posicionado o mais distal possível da valva tricúspide e próximo ao ápice ventricular. $\mathrm{O}$ eletrodo atrial foi fixado passivamente no apêndice atrial direito.

As medidas eletrofisiológicas foram realizadas considerando-se como satisfatórias: onda $\mathrm{P}$ superior a 1,2 $\mathrm{mV}$, limiar de estimulação atrial inferior a $1,0 \mathrm{~V}$; onda $\mathrm{R}$ superior a $4,0 \mathrm{mV}$, limiar de estimulação ventricular inferior a $0,8 \mathrm{~V}$ e a fixação adequada dos eletrodos foi assegurada por manobras respiratórias. Os eletrodos foram fixados junto ao acesso venoso e conectados ao gerador de pulso DDD ou DDD-R. O gerador foi introduzido na bolsa subcutânea da região peitoral e a incisão suturada.

\section{Cuidados no Pós-Operatório Imediato}

Após o implante do marcapasso, o aparelho foi programado no modo DDD. A programação objetivou uma freqüência de pulso capaz de realizar captura consistente do átrio e de um intervalo átrioventricular reduzido para obter o menor GVSVE, conforme a ecocardiografia Doppler. O programa do gerador ocorreu na dependência do modelo do gerador de pulsos DDD utilizado com ou sem resposta de freqüência.

Para os pacientes que implantaram o gerador DDD convencional o marcapasso foi reprogramado ainda na internação hospitalar, até 2 dias após o implante, durante a avaliação ecocardiográfica bidimensional com Doppler. Buscou-se obter a menor obstrução na VSVE mediante apropriado ajuste do marcapasso: operação em modo DDD, em freqüencia que resultasse em comando elétrico atrial e ventricular (freqüência basal de $70 \mathrm{ppm}$ a $85 \mathrm{ppm}$ ) e um intervalo A-V reduzido (variável entre $50 \mathrm{~ms}$ a $120 \mathrm{~ms}$ ). Para 
cada intervalo A-V programado $(150 \mathrm{~ms}, 100 \mathrm{~ms}, 75-70 \mathrm{~ms} \mathrm{e}$ $50 \mathrm{~ms}$ ). Foi aguardado o período de 5 minutos para a avaliação da obstrução na VSVE pelo ecocardiograma. O intervalo $\mathrm{A}-\mathrm{V}$ correspondente à menor obstrução foi programado no gerador.A alta hospitalar ocorreu no segundo dia após a cirurgia, com a medicação cardiovascular mantida.

\section{Seguimento dos Pacientes}

A medicação cardiovascular foi mantida após o ajuste do marcapasso. Os pacientes foram orientados para retornar 1,3, 6 meses e 1 ano para novas verificações clínicas e para exames ecocardiográficos ou, se indicado, ajuste do marcapasso. Avaliações foram programadas após o primeiro ano, ou de acordo com as necessidades clínicas e do gerador.

\section{Análise estatística}

Os valores do GVSVE foram apresentados como média \pm desvio-padrão. Para a comparação entre valores de GVSVE pré e pós implantação de marcapasso foi utilizado o teste $\mathrm{t}$ de Student para amostras dependentes. O nível de significância considerado foi de 0,05 . Foi utilizado o SPSS v. 8.0 (Statistical Package for Social Sciences) para as análises estatísticas realizadas.

\section{RESULTADOS}

\section{Mortalidade}

$\mathrm{Na}$ cirurgia e no período pós-operatório imediato, não foram registrados óbitos. Um paciente masculino de 46 anos faleceu com quadro de morte súbita no décimo segundo mês após o implante do marcapasso, perfazendo mortalidade tardia de 2,38\%.

O GVSVE deste paciente no pré-operatório era de 113 $\mathrm{mmHg}$, depois baixou para $80 \mathrm{mmHg}$ no acompanhamento imediato e era de, aproximadamente $46 \mathrm{mmHg}$ no momento do óbito.

\section{Morbidade}

No pré-operatório imediato e no período pós-operatório tardio, um paciente (2,38\% dos casos) teve estimulação diafragmática pela posição do eletrodo atrial. Essa complicação não foi solucionada por nova programação e foi indicada uma reposição para o eletrodo atrial. Após a nova cirurgia, o paciente vem evoluindo adequadamente.

\section{Evolução Clínica}

A maioria dos pacientes mostrou melhora clínica, exceto em 5 pacientes que não diminuiram o GVSVE. Na última avaliação, 29 pacientes estavam em classe funcional I, 7 pacientes na classe funcional II e 1 paciente na classe funcional III por evolução da insuficiência mitral. A redução da sintomatologia também foi importante, sendo que 29 pacientes estão assintomáticos, e 10 pacientes referem angina leve e negam dispnéia e tonturas.

A medicação foi suspensa em 3 pacientes $(7,14 \%$ dos casos) devido à melhora dos sintomas e ao aumento da capacidade física aos exercícios. A medicação foi suspensa naqueles casos onde o implante de marcapasso reduziu para zero ou para próximo de zero o GVSVE, e não houve necessidade de continuação do uso de drogas para manter o GVSVE baixo. Em 38 pacientes (90,4\%), foi necessário o aumento ou a mudança do esquema terapêutico, visando reduzir a frequiência cardíaca para assegurar o controle atrial pelo marcapasso, para diminuir a angina ou para reduzir ainda mais o GVSVE. Nesses casos, as drogas usadas foram beta-bloqueadores e verapamil em associação ou isoladamente.

Em 4 pacientes (9,5\% dos casos) pacientes foi indicada a ablação do ramo septal responsável pela irrigação da região hipertrofiada.

O GVSVE era de 91,3 $\pm 30,55$ imediatamente antes da programação, tendo sido reduzido ao valor de 45,2 $\pm 22,84$ $\mathrm{mmHg}$ após o primeiro ajuste do marcapasso. A redução do GVSVE foi observada em 92,9\% dos pacientes após o implante do marcapasso. Em 13 pacientes, o grau de obstrução tornou-se igual ou inferior a $30 \mathrm{mmHg}$. Em 12 pacientes, o GVSVE manteve entre $31 \mathrm{mmHg}$ e $50 \mathrm{mmHg}$ e em 17 pacientes, o GVSVE permaneceu superior a $51 \mathrm{mmHg}$. No acompanhamento, a reprogramação do marcapasso foi executada em 42 pacientes, e foram modificados parâmetros, como a freqüência cardíaca e o intervalo átrio-ventricular para diminuir o GVSVE. A média do GVSVE, na última avaliação foi de $34,7 \pm 23,03 \mathrm{mmHg}$ representando uma redução significativa quando comparada aos valores préoperatórios (teste t de Student para amostras dependentes; $\mathrm{p}<0,001)$.

Quanto à redução do GVSVE, classificaram-se os pacientes dos resultados: satisfatório (grupo I); aceitável (grupo II) e insatisfatório (grupo III). Observou-se a seguinte distribuição: 22 pacientes no grupo I, 10 pacientes no grupo II e 9 pacientes no grupo III (Figura 5).

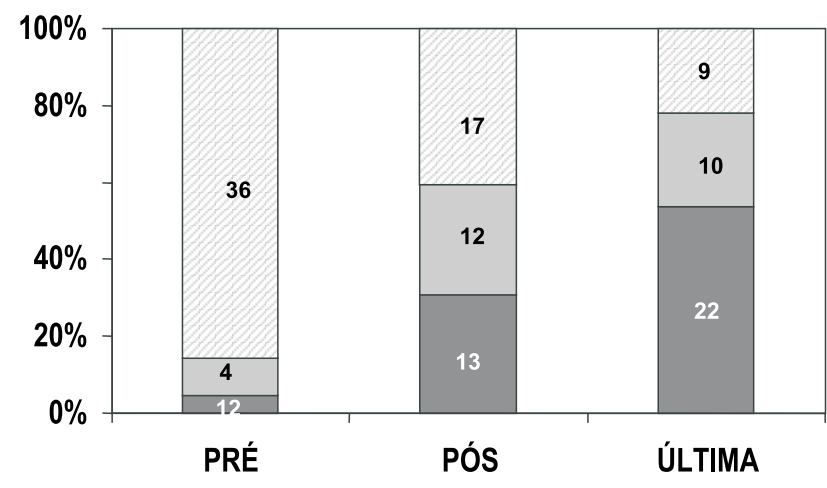

$\square$ até $30 \mathrm{mmHg} \square 31$ a $50 \mathrm{mmHg} \square$ acima de $50 \mathrm{mmg}$

Fig. 5 - Gradiente na via de saída do ventrículo esquerdo (GVSVE) de acordo com o momento cirúrgico 
Em alguns casos, o GVSVE era muito alto, e a redução foi dramáticajá nos primeiros momentos após o implante (Figura 5).

\section{COMENTÁRIOS}

A miocardiopatia hipertrófica obstrutiva tem apresentação heterogênea e manifestações clínicas decorrentes dessa apresentação, daí a dificuldade do tratamento, caracterizado por interações fisiopatológicas complexas entre a massa ventricular esquerda, o movimento sistólico anterior da valva mitral, as pressões, a disfunção diastólica e a isquemia do miocárdio.

Até há poucos anos, o tratamento indicado para os pacientes com miocardiopatia hipertrófica, resistentes à terapia medicamentosa ou que não a toleravam consistia na cirurgia, que tinha duas opções a apresentar: a miomectomia septal ou a substituição da valva mitral, com um índice de morbidade e de mortalidade inerente às técnicas. Mais recentemente, foi introduzida a ablação do ramo septal da artéria descendente anterior, pela injeção de álcool absoluto com o objetivo de produzir um infarto miocárdico septal alto com redução da massa ventricular obstrutiva. Os inconvenientes da técnica são a indução de um infarto com as complições inerentes precoces, e os bloqueios trifasciculares que determinam a implantação de marcapasso em uma porcentagem considerável dos pacientes. Os resultados a longo prazo seguem sendo uma incógnita. Ainda é objeto de controvérsia a forma de se adquirir experiência para a ablação dos ramos septais em pacientes referidos para o procedimento, já que o extravasamento do álcool para outros ramos coronarianos pode ser extremamente deletério, ou mesmo fatal a curto e a médio prazos.

Recentemente, tem havido maior interesse na aplicação da tecnologia do marcapasso para os pacientes que não têm resposta adequada aos medicamentos e que não serão submetidos às terapêuticas cirúrgicas convencionais.

Os primeiros conceitos foram propostos na Alemanha (19) e em seguida na França. ${ }^{(23)}$. Em 1988, McDonald ${ }^{(24)}$ descreveu os benefícios sintomáticos associados ao marcapasso de dupla câmara em 11 pacientes. Em 1992, investigadores suíços ${ }^{(25)}$ demonstraram redução parcial do gradiente da via de saída com o uso do marcapasso de forma temporária ou permanente, bem como da melhora subjetiva dos sintomas. No trabalho de Fananapazir, ${ }^{(26)} 84$ pacientes foram avaliados e vários benefícios foram alcançados em pacientes submetidos ao implante do marcapasso, tais como: melhora dos sintomas com diminuição da hipertrofia ventricular e diminuição do GVSVE após um período de acompanhamento de 2 anos.

Estudos recentes ${ }^{(27,28)}$ introduziram a eletroterapia para a miocardiopatia refratária através da colocação de um marcapasso seqüencial DDD com intervalo átrio-ventricular curto, quando o eletrodo atrial preserva a contração atrial que é de extrema importância para a manutenção do débito cardíaco nos pacientes com disfunção diastólica e, pelo eletrodo ventricular que é o responsável pela desestruturação da contratilidade septal, originando uma diminuição significativa do gradiente na via de saída do ventrículo esquerdo. Então, naqueles pacientes em classe III e IV da NYHA refratários ao tratamento clínico, pôde-se aventar a possibilidade da implantação do marcapasso, que, dentre as opções disponíveis é o procedimento menos invasivo e potencialmente menos deletério. Uma das dificuldades encontradas é a seleção dos pacientes que irão se beneficiar com a eletroterapia já que não existe correlação entre os parâmetros clínicos dos pacientes e os diferentes resultados. Outra limitação da terapêutica é a presença de diferentes gradientes para o mesmo paciente em situações diversas. Como a maior parte dos pacientes tem um gradiente importante em repouso e, esses foram os maiores beneficiados, algumas conclusões podem ser alcançadas, mas de forma alguma, é o corolário deste estudo. A perda de consciência ou a síncope estão associadas à maior mortalidade ${ }^{(29)} \mathrm{e}$, no grupo dos pacientes tratados, houve uma diminuição dos episódios, o que seria tendência para a diminuição da morte súbita durante o período de acompanhamento.

Existem razões para as poucas correlações entre as evoluções aguda e crônica do marcapasso: 1) as pequenas mudanças no rendimento cardíaco podem produzir alterações no gradiente da via de saída do ventrículo esquerdo, então, se o marcapasso aumenta o rendimento cardíaco, o gradiente pode não se alterar ou até aumentar; 2) as mudanças adaptativas hemodinâmicas, em resposta ao estímulo DDD crônico, contribuem significativamente para o sucesso da terapia.

Alguns pacientes têm comportamento paradoxal após o implante do marcapasso, ou seja, pacientes que têm importante redução do gradiente no pós-operatório imediato podem voltar a ter aumentos de gradiente a médio e longo prazos e pacientes que não tiveram mudanças significativas de modo agudo podem ter diminuição do gradiente de modo lento e progressivo

Em alguns pacientes, a dificuldade de comando é agravada pela condução lenta intra-atrial, o que pode ser mostrado pelas ondas $\mathrm{P}$ prolongadas no ECG de superfície. A programação dos intervalos $\mathrm{A}-\mathrm{V}$, que são muito curtos nesses pacientes, para assegurar a captura ventricular, pode levar à contração simultânea do ventrículo e do átrio esquerdos. A contração do átrio esquerdo com a valva mitral fechada pode resultar em congestão pulmonar, diminuir o enchimento diastólico do ventrículo esquerdo e, então, de novo, piorar a obstrução da via de saída do ventrículo esquerdo.A ablação por radiofreqüência do nó $\mathrm{A}-\mathrm{V}$ pode assegurar maior captura ventricular e permitir a seqüência da contração átrio-ventricular. Embora o procedimento torne o paciente dependente do marcapasso, pode se justificar pela diminuição do gradiente com perspectiva de maior benefício e prescindir de uma cirurgia cardíaca.

$\mathrm{O}$ seguimento a longo prazo tem mostrado que pode 
haver diminuição da espessura da parede do septo interventricular em alguns pacientes. Nos pacientes onde houve a diminuição da espessura do septo interventricular, foi observada a melhora dos sintomas. Se os achados forem confirmados, abre-se a perspectiva de redução da massa ventricular em crianças e em adolescentes atenuando e prevenindo a hipertrofia muscular no período de maior crescimento corporal, no septo e no ventrículo esquerdo.

Num estudo de Fananapazir ${ }^{(20)}, 95 \%$ dos pacientes melhoraram sua classe funcional de $3,4 \pm 0,5$ para $1,7 \pm 0,7 \mathrm{e}$ foram acompanhados de melhora na sintomatologia clínica, com diminuição da congestão pulmonar. Na série, $70 \%$ dos pacientes com angina passaram para $25 \%$ dos casos. Nos 15 pacientes com síncope de repetição, apenas um teve novo episódio após 9 meses de seguimento.

A instituição de um tratamento traz efeitos fisiológicos e psicológicos, os quais estão inter-relacionados no paciente. $\mathrm{O}$ efeito placebo pode trazer um bom resultado e agir em conjunto com o tratamento ativo, influenciando o resultado da terapêutica. Os pacientes que mantêm a terapêutica medicamentosa podem apresentar um efeito aditivo do marcapasso sobre a condução cardíaca no ventrículo direito e, desta forma, melhorar a sístole que é modificada com drogas de inotropismo negativo. Se, por acaso, houver a descontinuação das drogas, o paciente não estará sofrendo dos efeitos colaterais das mesmas, trazendo com isso alívio importante sob os pontos de vista fisiológico e psicológico.

Nenhum dos procedimentos mostrou-se eficaz para todos os pacientes e tampouco preveniu a morte súbita. O objetivo primário foi aliviar os sintomas e melhorar a capacidade e a tolerância aos exercícios, sendo que os procedimentos não se excluem e podem ser complementares, por exemplo; o paciente que não se beneficia com o marcapasso pode ser submetido à cirurgia cardíaca, e os pacientes que seguem sintomáticos ou complicam com bloqueio átrio-ventricular total ou com bloqueio trifascicular irão se beneficiar com o implante do marcapasso.

Essa filosofia foi incorporada ao presente estudo e quatro pacientes do grupo foram referidos para a ablação septal com álcool absoluto e tiveram boa evolução com diminuição doGVSVE.

Com o crescimento gradual e irreversível do grau de conhecimento do código genético dos familiares comprometidos, seria factível supor que a terapêutica com marcapasso fosse indicada o mais precocemente possível e posta ao alcance daqueles pacientes com um risco maior de desenvolver sintomatologia incapacitante num futuro próximo.

A identificação genotípica para os pacientes com risco de desenvolver a doença antes e independentemente da presença de sintomas ou do desenvolvimento da hipertrofia oferece informações valiosas para a prevenção da morte súbita e da insuficiência cardíaca. A possibilidade de identificar e de separar pacientes com mutações de alto risco daqueles sem a mutação ou de baixo risco oferece dados essenciais para o médico que determinar condutas para crianças e indivíduos assintomáticos, da mesma forma que permitirá orientar diferentes terapêuticas para grupos específicos de pacientes.

Existem alguns grupos de pacientes com características especiais, como os pacientes com bloqueio do ramo esquerdo, onde a ativação elétrica anormal do septo interventricular poderia comprometer o resultado da eletroterapia. Nesse grupo de pacientes, as novas experiências com eletrodos, em 3 ou 4 câmaras cardíacas, talvez, fizessem a diferença na qualidade da estimulação átrio-ventricular com a ajuda da ecocardiografia, sincronizando a atividade dos átrios e ventrículos. Nesse, e, talvez, em outros grupos especiais, a ajuda do eletrofisiologista pode ser fundamental tanto na detecção de vias anômalas quanto na ablação de feixes que possam levar a contrações precoces, prejudicando a melhor seqüência de ativação átrio-ventricular.

Um outro benefício adicional que pode ser aventado é o implante do marcapasso em crianças e em adolescentes durante o período de crescimento rápido, o que parece ter relação com o crescimento assimétrico do coração. Nestes pacientes, eletrodos e geradores específicos seriam implantados com o objetivo de diminuir o crescimento ventricular esquerdo e, mantendo o sincronismo átrioventricular alcançariam um benefício adicional diminuindo a chance do aparecimento de arritmias atriais e ventriculares. Os sistemas usados hoje em dia nada mais são do que adaptações de marcapassos para o tratamento de bradicardia e, talvez, não supram todas as necessidades de uma doença com a fisiopatologia ainda pouco conhecida.

Em todas as séries revisadas, houve uma melhora significativa dos sintomas e na diminuição do GVSVE. Os resultados alcançados também mostram que a melhora não acontece para todos os pacientes e, que pode, inclusive, haver piora da sintomatologia ${ }^{(30)}$. A grande vantagem para esse tipo de problema é de que o marcapasso pode ser desligado a qualquer momento, e que suas funções podem ser alteradas sem necessidade de nova cirurgia, em contraste com qualquer outro procedimento elencado anteriormente $^{(31)}$.

Na experiência aqui apresentada, a redução do GVSVE foi, aproximadamente de $57 \%$ do inicial, o que se aproxima da experiência de outros centros como a descrita por SADOUL et. al. ${ }^{(32)}$ que mostrou ter havido $48 \%$ de redução do GVSVE após o implante.

Dos procedimentos atualmente disponíveis, a eletroterapia pode ser aplicada em um maior número de centros com menores recursos, além da capacidade de readequação dos parâmetros em qualquer lugar, e a qualquer momento. É factível supor que novas tecnologias possam reconhecer estados diferentes de contratilidade cardíaca em momentos distintos e possam haver alterações, esquerdo dinâmicas durante as possíveis mudanças de gradiente da via de saída do ventrículo. 


\section{CONCLUSÕES}

Os resultados obtidos nessa série mostraram que pacientes com miocardiopatia hipertrófica obstrutiva pouco responsivos à terapêutica farmacológica e com gradientes elevados acima de $50 \mathrm{mmHg}$ beneficiam-se com o estímulo do marcapasso cardíaco em modo DDD com PR curto e ajustado à cada paciente de modo que o estímulo ventricular seja sempre mediado pelo marcapasso.

O implante do marcapasso seqüencial de dupla câmara é efetivo para a melhora dos sintomas como: palpitações, angina, dispnéia e estados sincopais. A melhora dos sintomas está relacionada à diminuição do gradiente na via de saída do ventrículo esquerdo. Foi observado que os benefícios se mantêm com o passar do tempo nos pacientes que, desde o início, melhoraram e, em alguns que não tiveram benefício imediato.

Durante o acompanhamento, ocorreu importante redução no gradiente sistólico máximo na via de saída do ventrículo esquerdo pelo ecocardiograma com Doppler. A melhora hemodinâmica foi acompanhada pela significativa queda do GVSVE imediatamente após o implante do marcapasso e, naqueles pacientes que não tiveram benefício imediato, as reduções do GVSVE foram obtidas com um reajuste posterior. Nos pacientes que não tiveram queda significativa após as primeiras programações, apesar da medicação e das reprogramações, os sintomas persistiram e, nesse grupo, está incluído o paciente que evoluiu para morte súbita. Não existe diferença no grau de benefício entre os sexos, isto é, ambos se beneficiaram igualmente.

Em alguns pacientes, a eletroterapia para a $\mathrm{MHO}$ permite que a melhora sintomática e da classe funcional seja acompanhada pela diminuição dos medicamentos, ou mesmo, da abolição dos mesmos sem perda dos benefícios conseguidos.

Em todas as idades, os pacientes tiveram benefício semelhante já com uma queda importante do GVSVE no primeiro mês, benefício este que se mantém, na maioria dos casos, independente da idade.

A presente experiência pode ser estendida a outros centros pela facilidade e pela reprodutibilidade da técnica, aumentando a tolerância aos esforços e a melhora dos sintomas na maioria dos pacientes.

\section{REFERÊNCIAS BIBLIOGRÁFICAS}

1. Liouville H - Rétrécissement cardiaque sous aortique. Gaz Med Paris 1869;24:161-163.

2. Hallopeau L - Rétrécissement ventriculo-aortique. Gaz Med Paris 1869;24:683-684.

3. Schmincke A - Ueber linksseitige muskuloe Conusstenosen. Dtsch Med Wochenschr 1907;33:2082.
4. Rock RC - Functional obstrution of the left ventricle. Guys Hosp Rep 1957;106:221-238.

5. Maron BJ, Gardin JM, Flack JM, Gidding SS, Kurosaki TT, Bild DE - Prevalence of hypertrophic cardiomyopathy in a general population of young adults: echocardiographic analysis of 4111 subjects in the CARDIA Study. Circulation 1995;92:785-9.

6. Teare RD - Asymmetrical hypertrophy of the heart in young adults. Br Heart J 1958;20:1-8.

7. Savage DD, Castelli WP, AbbottRD, Garrison RJ, Anderson SJ, Kannel WB, Feinleib M - Hypertrophic cardiomyopathy and it $\mathrm{s}$ markers in the general population: The great masquerader revisited: The Framingham Study. J Cardiovasc Ultrason 1983;2:41-47.

8. Ishanov A, Okamoto H, Yoneya $\mathrm{K}$, et al. - Angiotensinogen gene polymorphism in Japanese patients with hypertrophic cardiomyopathy. Am Heart J 1997;133:184-9.

9. Lechin M, Quiñones MA, Omran A, et al - Angiotensin-I convertin enzyme genotypes and left ventricular hypertrophy in patients with hypertrophyc cardiomyopathy. Circulation 1995;92:1808-12.

10. Iwai N, Ohmichi N, Nakamura Y, Kinoshita M - DD genotype of the angiotensin-converting gene is a risk factor for the left ventricular hypertrophy. Circulation 1994;90:2622-8.

11. Spirito P, Seidman CE, Mc Kenna WJ, Maron BJ - The management of hypertrophic cardiomyopathy. N Engl J Med 1997;33:775-85

12. Davies MJ, McKenna WJ - Hypertrophic cardiomyopathy an introduction to pathology and pathogenesis. Br Heart J 1994;72 (Suppl):S2-S3

13. McIntosh CL, Maron BJ - Current Operative Treatment of Obstructive Hypertrophic Cardiomyopathy. Circulation 1988;78:487-495.

14. Louie EK, Maron BJ. Hypertrophic cardiomyopathy with extreme increase in left ventricular wall thickness: functional and morphologic features and clinical significance. J Am Coll Cardiol 1986;8:57-65

15. Spirito P, Chiarella F, Carratino L, et al. - Clinical course and prognosis of hypertrophic cardiomyopathy in an outpatient population. N Engl J Med 1989;320:749.

16. Frank S, Braunwald E - Idiopathic hypertrophic subaortic stenosis. Clinical analysis of 126 patients with emphasis on the natural history. Circulation 1968;37:759.

17. Brigden W - Hypertrophic cardiomyopathy. Br Heart J 1987;58:229.

18. Frenneaux MP, Porter A, Caforio AL, et al. - Determinants of exercise capacity on hypertrophic cardiomyopathy. J Am Coll Cardiol 1989;13:1521. 
19. Hassenstein P, Storch H-H, Schmitz W - Erfahrungen mit schrittmacherdauer behandlung bei patienten mit obstruktiver kardiomyopathie. Results of eletrical pacing in patients with obstrutive cardiomyopathy. Thoraxchirurgie 1975;23:496-8.

20. Fazanapazir 1, Cannon RO, Tripodi D, Panza JÁ - Impact of dual chamber permanent pacing in pacients with obstructive hypertrophic cardiomyopathy with symptons refractory to verapamil and beta-adrenergic blocker therapy. Circulation 1992;85:2149-61.

21. Sadoul N,Simon JP, de Chillou C, et al. Long-term dual-chamber pacing improves hemodynamic function in patients with obstrutive hypertrophic cardiomyopathy. Pace 1993; 16(5parte 2):1120.

22. Wigle ED, Sasson Z, Henderson MA, et al. - Hypertrofic cardiomyopathy. the importance of the site and the extent of hypertrophy. a review. Prog Cardiovasc Dis 1985;28:1-83.

23. Duport G, Valeix B, Lefevre J, et al. - Interet de la stimulation ventriculaire droite permanente dans la cardiomyopathie obstructive. Nouv Presse Med 1978;32:2868-9.

24. McDonald K, McWilliams E, O'Keefe B, et al. - Functional assessment of patients treated with permanent dual chamber pacing as a primary treatment for hypertrophic cardiomyopathy. Eur Heart J 1988;9:893-8.

25. Jeanrenaud X, Goy J-J, Kappenberger L - Effects of dualchamber pacing in hypertrophic obstructive cardiomyopathy. Lancet 1992;339:1318-23.
26. Fananapazir L, Epstein ND, Curiel RV, Panza JA, Tripodi D, McAreavey D - Long term results of dual-chamber (DDD) pacing in obstructive hypertrophic cardiomyopathy. Circulation 1994;90:2731-42.

27. McDonald K, Maurer B - Permanent pacing as treatment for hypertrophic cardiomyopathy. Am J Cardiol 1991;68:10810.

28. Fananapazir L, Srinivas V, Bennett DH - Comparison of resting hemodynamics indices and exercise performance during atrial syncronized and assyncronous ventricular pacing. Pace 1983;6:202-9.

29. Maron BJ, Fananapazir L - Sudden cardiac death in hypertrophic cardiomyopathy. Circulation 1992;85(suppl I):I57-I-63.

30. Nishimura RA, Trusty JM, Hayes DL, et al - Dual-Chamber pacing for Hypertrophic Cardiomyopathy: A Randomized, Double-Blind, Crossover Trial. J Am Coll Cardiol 1997;29:43541.

31. Sant $₫$ Anna JR, Prati R, Hutten H, et al - Ventricular evoked response in patients with hypertrophic obstructive cardiomyopathy treated with DDD pacing. Arq Bras Cardiol 1999;73(2):169-79.

32. Sadoul N, Simon JP, Chillou C, et al - Intérêts de la stimulation cardiaque permanente dans les myocardiopathies hypertrophiques et obstructives rebelles au traitement médical. Arch Mal Coeur 1994;87:1315-23. 